\title{
Serial prostate magnetic resonance imaging fails to predict pathological progression in patients on active surveillance
}

Danly Omil-Lima ${ }^{1}$; Albert Kim²; Ilon Weinstein ${ }^{3}$; Karishma Gupta ${ }^{1}$; David Sheyn ${ }^{1}$; Lee Ponsky ${ }^{1,4}$

${ }^{1}$ Urology Institute, University Hospitals Cleveland Medical Center, Cleveland, Ohio; School of Medicine, Case Western Reserve University, Cleveland, OH, United States; ${ }^{2}$ Division of Urology and Urologic Oncology, City of Hope Comprehensive Cancer Center, Duarte, CA, United States; ${ }^{3}$ School of Medicine, Case Western Reserve University, Cleveland, ON, United States; ${ }^{4}$ Case Comprehensive Cancer Center, Case Western Reserve University School of Medicine, Cleveland, OH, United States

Cite as: Omil-Lima D, Kim A, Weinstein I, et al. Serial prostate magnetic resonance imaging fails to predict pathologic progression in patients on active surveillance. Can Urol Assoc J 2022 February 28; Epub ahead of print. http://dx.doi.org/10.5489/cuaj.7541

Published online February 28, 2022

Appendix available at cuaj.ca

Corresponding author: Dr. Lee Ponsky, University Hospitals Cleveland Medical Center, Case Western Reserve University School of Medicine, Cleveland, $\mathrm{OH}$, United States;

Lee.Ponsky@uhhospitals.org

$* * *$

\section{Abstract}

Introduction: Limited data guide urological practice when employing prostate magnetic resonance imaging (MRI) in active surveillance protocols. To determine the ability of prostate MRI to predict pathological progression in active surveillance patients, we correlated findings of serial MRI with results of surveillance biopsies.

Methods: Patients on active surveillance with $\geq 2$ prostate MRI and $\geq 2$ prostate biopsies were included. Prostate Imaging-Reporting and Data System (PI-RADS) score upgrade, as assigned by experienced radiologists, was used to assess the ability of imaging to predict pathological biopsy progression. Imaging test statistics and the odds ratio of pathological progression according to MRI upgrade were calculated.

Results: Of 121 patients meeting criteria, 36 (30\%) demonstrated MRI upgrade. Biopsy progression was noted in 55 patients $(46 \%)$. Of these, 20 patients $(37 \%)$ had biopsy progression predicted by MRI upgrade, while the remaining $(\mathrm{n}=35)$ had no lesion upgrade on prostate MRI. Conversely, among those with no biopsy progression $(n=66), 16$ patients $(24 \%)$ had a false- 
positive upgrade on serial MRI. We report a sensitivity and specificity of MRI change for pathological progression of $36 \%$ and $76 \%$, respectively. Although MRI change was associated with a positive predictive value of $56 \%$ for pathological progression, patients with a highsuspicion lesion (PI-RADS $>3$ ) at any time were more likely to experience disease progression, (odds ratio 3.3, 95\% confidence interval 1.6-8.0, $\mathrm{p}<0.01$ ).

Conclusions: Given its modest sensitivity/specificity, serial prostate MRI should be used judiciously as a surveillance tool. However, when prostate MRI demonstrates a PI-RADS $>3$ lesion, a high index of suspicion should be maintained, as these patients are more likely to progress on active surveillance.

\section{Introduction}

Prostate specific antigen (PSA)-based screening has improved the early detection of prostate cancer (PCa), resulting in more men being diagnosed and treated. ${ }^{1}$ However, the majority of screen-detected $\mathrm{PCa}$ is low-risk and prospective cohort studies have shown that active surveillance (AS) is a safe option for initial management given the low likelihood of progression to metastatic disease and low cancer-related mortality. ${ }^{2,3}$

There are currently no consensus regarding the optimal protocol for AS. While most protocols include a combination of PSA testing, prostate biopsy, and digital rectal examination, others have incorporated prostate genetic biomarkers, testing for different PSA isoforms, as well as imaging utilizing multi-parametric prostate MRI (mpMRI). ${ }^{4}$ Indeed, current National Comprehensive Cancer Network (NCCN) guidelines for active surveillance suggest consideration of mpMRI, along with prostate biopsy, no more often than every 12 months. ${ }^{5}$ Although these guidelines suggest a minimum time interval between imaging, they do not provide guidance on optimal use of MRI and how imaging results should influence treatment decisions, including surveillance biopsy and discontinuation of AS. Given this ambiguity, some have incorporated mpMRI in a serial fashion to allow for increased intervals between biopsies and to mitigate patient anxiety. ${ }^{6}$ While foregoing prostate biopsy would likely result in greater compliance and reduced complications, the American Urological Association Multiparametric Prostate MRI Consensus Panel deems current data to be insufficient regarding repeat mpMRI without a prostate biopsy for monitoring men on AS. ${ }^{7,8}$

Here, we assess the utility of repeat mpMRI in the management of PCa patients on AS based on the ability of mpMRI to predict pathologic progression. We hypothesize that serial MRI results do not provide additional information to impact decision making in AS.

\section{Methods}

Retrospective chart review was conducted to identify PCa patients on AS having undergone $\geq 2$ mpMRIs at University Hospitals Cleveland Medical Center from 2012-2020. Patient 
characteristics were compared using descriptive statistics. Per NCCN guidelines, low-risk PCa was defined as T1-T2a disease, Gleason Grade Group (GGG) 1 and PSA $<10 \mathrm{ng} / \mathrm{mL}$, while favorable-intermediate risk was defined as GGG1 or GGG2, PSA at diagnosis between 10$20 \mathrm{ng} / \mathrm{mL}$ and $<50 \%$ biopsy cores positive. Records were further screened to select patients with $\geq 2$ prostate biopsies during their time on AS. Acceptable prostate biopsies were heterogeneous, including standard 12-core biopsies and MRI-fusion biopsies (UroNav and In-Gantry). All MRIs were read and scored by experienced, board-certified radiologists using the Prostate Imaging Reporting and Data System (PI-RADS) version 2, where applicable. ${ }^{9}$ Prostate mpMRI obtained prior to 2016 (release of PI-RADS v2 recommendations) were re-reviewed and scored accordingly by radiologists. Patients whose MRI could not be assigned a PI-RADS score were excluded. In case of multiple PI-RADS lesions, the lesion with the highest score was used. MRI lesion upgrade was defined as an increase in PI-RADS score of the previously existing index lesion or the appearance of a new high-suspicion lesion, defined as PI-RADS $>3$. Pathologic progression was defined as increase in GGG score on subsequent prostate biopsy, as assigned by board certified genitourinary pathologists.

Test statistics including sensitivity, specificity, positive predictive value (PPV), and negative predictive value (NPV) were calculated based on change between the first and second MRI in relation to pathological progression on prostate biopsy. The association between mpMRI and biopsy progression was examined using Fisher's exact test of contingency data, analyzed in Prism 9 (GraphPad Software Inc., San Diego, CA) and STATA16 (StataCorp LLC, College Station, Texas). In addition to measuring the risk of pathological progression based on mpMRI upgrade, we also calculated the odds ratio (OR) of biopsy progression with a high-suspicion lesion (PI-RADS $>3$ ) present at any time on AS (i.e. regardless of upgrade). In order to determine whether results of mpMRI influenced treatment change, we compared the average time on active surveillance amongst patients with and without mpMRI upgrade using a two-tailed unpaired $t$ test. Lastly, a multivariable logistic regression model was fit to determine if change in PI-RADS score on MRI could predict biopsy progression. An alpha level of 0.05 was chosen for statistical significance.

\section{Results}

A total of 121 patients meeting study criteria were identified. Patient demographics are demonstrated in Supplemental Table 1, with no statistically significant differences in patient characteristics amongst patients with and without MRI upgrade. Data in Supplemental Table 2 demonstrate patient demographics according to the presence pathologic progression. Patients who experienced pathologic progression differed from patients without progression by median PSA at diagnosis only (median $5.8 \mathrm{vs} 4.7 \mathrm{ng} / \mathrm{ml}$, respectively, $\mathrm{p}<0.05$ ) .

Figure 1A demonstrates the percentage of mpMRI change. While $30 \%$ of repeat mpMRI demonstrated PI-RADS upgrade, most studies either remained the same or demonstrated regression/resolution of the index lesion. Among 36 patients who experienced upgrade on 
mpMRI, 20 patients (56\%) also demonstrated pathologic progression on biopsy, Figure 1B. These patients were noted to progress to Gleason $3+4=7(n=15)$, and Gleason $4+3=7(n=4)$ disease, with one patient deemed to progress to Gleason $3+3=6$ with higher volume of disease on biopsy.

The sensitivity and specificity of MRI upgrade for detecting pathologic progression were 36.4\% (95\% CI 23.6\%-49.0\%) and 75.8\% (65.4\%-86.0\%), respectively (Figure 2A). The PPV and NPV of mpMRI upgrade for pathologic progression were 55.6\% (39.3\%-71.7\%) and 58.8\% (48.3\%-69.2\%) respectively. Figure 2B demonstrates the relationship between MRI upgrade and biopsy progression. The likelihood of biopsy progression was not statistically more likely in cases where MRI upgrade was present, owing to an OR of 1.786 (95\% CI 0.8 to 3.8), $\mathrm{p}=0.16$.

Patients with a high-suspicion lesion on mpMRI at any time during AS were more likely to experience biopsy progression, OR 3.3 (95\% CI 1.6-8.0), $\mathrm{p}<0.01$. The sensitivity and specificity of a single high-suspicion lesion for predicting biopsy progression on AS was $78.1 \%$ (66.0-87.1\%) and 50.0\% (38.2-61.7\%) respectively. Using the presence of a high-suspicion lesion at any time as the screening tool, the number needed to screen to detect biopsy progression on subsequent surveillance prostate biopsy was 3.3 men (95\% CI 2.2-9.6).

Supplemental Figure 1 demonstrates no statistically significant differences in time on active surveillance between men with and without MRI upgrade, $p=0.19$.

On univariate regression, MRI upgrade did not predict biopsy upgrade (OR 1.8, 95\% CI 0.8-3.9; $\mathrm{p}=0.15$ ). On multivariate analysis including other factors such as age, PSA at diagnosis, follow-up time, grade group at diagnosis, risk category at diagnosis, prostate volume, number of MRIs, time between MRIs, and number of prostate biopsies, MRI upgrade did not predict biopsy progression (OR 2.5, 95\% CI 0.6-9.6; $\mathrm{p}=0.19$ ). Odds ratios for pathologic progression are detailed in Supplemental Table 5.

\section{Discussion}

AS involves monitoring patients closely in order to offer treatment within a window of curability. Traditional clinical risk factors (PSA, DRE, and prostate biopsy) have been relatively successful in assessing patient risk in AS. However, limited data guide urologic practice when employing mpMRI in AS protocols. Thus, our study specifically aimed to evaluate the utility of repeat/serial mpMRI in PCa patients on AS. We add several key findings to the paucity of literature in this regard.

First, our data demonstrates limited utility in repeating an mpMRI in AS patients, as most lesions do not change [81 of 121 patients (70\%) without upgrade]. In a study of 49 patients with low-risk prostate cancer and mpMRI at least six months apart, Felker et al. observed 39 of 49 patients $(80 \%)$ had no progression of mpMRI. Furthermore, most lesions (67\%) did not change in size. ${ }^{10}$ This yielded a sensitivity and specificity of mpMRI to be $37 \%$ and $90 \%$ respectively. Similarly, in a study of 144 patients on a unique AS protocol of initial and yearly follow-up mpMRI in lieu of biopsy, Habibian et al. demonstrated that only 14 of 144 patients $(10 \%)$ had 
upgrade on mpMRIs, with a median follow up of 48 months. ${ }^{11}$ In their study, only 7 of the 14 patients with MRI upgrade went on to obtain biopsy. Thus, the rate of missed progression is unknown.

When compared to other studies in the literature, the current study includes a larger patient cohort and incorporates biopsy findings in all patients. We demonstrate $63 \%$ of incident progression is missed with MRI alone, as shown in Supplemental Table 3. Indeed, several cases of pathologic progression would have been missed by MRI alone, including progression to highrisk disease (Gleason $4+4=8$ and $4+5=9$ ).

The question remains whether there is a high likelihood of pathologic progression if mpMRI upgrade is seen. In a cohort of 76 patients on AS for low-risk prostate cancer, Eineluoto et al. demonstrated that 33 of 76 patients (43\%) had mpMRI upgrade, leading to 27 patients $(82 \%)$ undergoing treatment change. ${ }^{12}$ In our study, mpMRI PI-RADS upgrade demonstrated a PPV of only 55.6\%. Interestingly, a drop in PPV of mpMRI was seen in the current study when including patients after 2017, suggesting that either increasing sample size or increasing experience with mpMRI interpretation may have led to a closer approximation of the true PPV of imaging, or fewer discrepancies in initial PI-RADS scoring. Regardless, we highlight the need for judicious interpretation of repeat mpMRI results in PCa patients on AS.

A second important observation of our study is that the presence of high-suspicion lesions at any time are associated with increased pathologic progression, OR 3.6 (95\% CI 1.68.0). In their AS cohort, Kornberg et al looked at the risk of biopsy progression in 169 patients low-risk patients with a single mpMRI. Their results showed PI-RADS scores of 5 vs 1-2 (HR $4.38,95 \%$ CI 2.36-8.16, $\mathrm{P}<0.01$ ) and 4 vs $1-2$ (HR 2.62, 95\% CI 1.45-4.76, P <0.01) were significantly associated with an increased risk of a biopsy progression. ${ }^{13}$ These results bolster the significance of previously described high sensitivity and positive predictive value of PI-RADS $4 / 5$ lesions for detecting clinically significant prostate cancer. ${ }^{14-16}$ In this respect, mpMRI is a useful tool in patient counseling and determining candidacy for AS.

Indeed, some have argued that the most important role of mpMRI is that of riskstratification to determine patient eligibility for AS at the onset of surveillance. ${ }^{17}$ Our data supports this, as use of the presence of a high-suspicion lesion at any time, rather than MRI change, improved the sensitivity of mpMRI for detecting biopsy progression. Indeed, the UK National Institutes of Health and Care Excellence already recommends mpMRI as part of their AS initiation protocol. ${ }^{18}$ Notably, men in our series were maintained on AS for the same median follow up time regardless of mpMRI upgrade (Supplemental Figure 1), suggesting that its use as a longitudinal benchmark was not predictive of treatment change.

Finally, given the inherent risk of infection as well as patient discomfort and anxiety, the question of whether mpMRI can replace prostate biopsy has been debated. ${ }^{19}$ Our data demonstrate currently available mpMRI technology cannot replace surveillance biopsy, as this would miss 35 of 55 patients (64\%) with biopsy progression that had no upgrade on mpMRI $(\mathrm{NPV}=58 \%$ ). In a recently published large meta-analysis of 7,321 patients, Sathianathen et al. 
reported a NPV of mpMRI for detecting clinically significant prostate cancer to be $86.8 \%{ }^{20}$ Despite the higher NPV, the authors recommended proceeding with caution, as a negative mpMRI still missed clinically significant cancer in 7-10\% of men not proceeding to biopsy. This sentiment largely agrees with prior studies that deem mpMRI not accurate enough to replace prostate biopsy. ${ }^{21}$ In this regard, it is important to keep in mind that higher estimates of mpMRI NPV previously reported refer to the use of this technology in a diagnostic capacity, amongst allcomers, rather than as a tool for surveillance, as described in the current study. In other words, the question of whether mpMRI can replace initial prostate biopsy may be entirely different from whether it can replace surveillance biopsies. Thus, while the thought of replacing prostate biopsy with mpMRI in AS is attractive for patient and physicians alike, our study and others demonstrate that mpMRI should not replace prostate biopsy in either setting.

We acknowledge this study is not without several limitations. Firstly, our study was retrospective. Future prospective, randomized studies are required to further assess the utility of MRI in AS. Secondly, our study had a limited sample size, and data were derived from a single institution. Using previously published rates of biopsy progression amongst men on $\mathrm{AS},{ }^{22}$ we performed post-hoc power analysis, which demonstrated $100 \%$ power for detection of disease progression at an alpha level of 0.05 given the current sample size (data not shown). Additionally, to our knowledge, the current series is one of the largest incorporating both serial MRI and prostate biopsy pathologic data for all patients in an AS setting. Thus, we feel confident that the results shown here reflect the true performance of mpMRI in AS.

Another important limitation of the current study relates to radiological interpretation. Given the retrospective nature and use of clinical data, radiologists were not blinded to patients' previous imaging. We are unable to determine the inter-radiologist variability when scoring mpMRIs as these radiology reports were read in a clinical setting. As such, we only have access to final reports (often read by one radiologist at a time), not the details of peer review. Additionally, we were unable to capture changes in the size of lesions and their ADC values. These variables have been associated with disease progression in prior studies and were unable to be incorporated into the current study, as these were not commonly reported in clinical radiology reports. Indeed, whereas other studies have used imaging performed under a research protocol not available to treating clinicians, ${ }^{23}$ the current study utilized clinical imaging reports accessible to any urologist. Given challenges and discrepancies in reporting, the concept of standardized reporting of mpMRI has been proposed and would pay special attention to include specific assessment of changes over time. ${ }^{24}$ Despite these limitations, dedicated GU radiologists at our institution have experience reading prostate mpMRI daily in practice and have published on this topic. Thus we have no reason to expect that experience or inter-observer variability play any greater role in determining the results of the current study when compared to others in the literature. 
We also recognize that mpMRI continues to be expensive in some hospital systems and some clinicians may not have access to mpMRI and/or an experienced radiologist to interpret them. Our hope is to provide information for urologists in these settings to determine best practices for obtaining surveillance MRI.

\section{Conclusions}

With modest sensitivity and specificity, results from serial prostate mpMRI in PCa patients on AS should be interpreted judiciously. Specifically, change on mpMRI is infrequent and patients experience pathologic progression that is missed by serial imaging. Certainly, a repeat mpMRI should not replace biopsy in men on AS, as a high proportion of clinically significant disease will be missed.

Importantly, if mpMRI demonstrates a PI-RADS $>3$ lesion at any point, a high index of suspicion should be maintained, as these patients are more likely to have pathologic. In this regard, information gleaned from mpMRI can be used to risk stratify patients and confirm candidacy for AS. However, once a high-suspicion lesion has been identified, the benefit of further imaging is questionable.

Although data clearly support a role for MRI in aiding diagnosis of clinically significant prostate cancer, the benefit of serial imaging in a surveillance capacity requires further research.

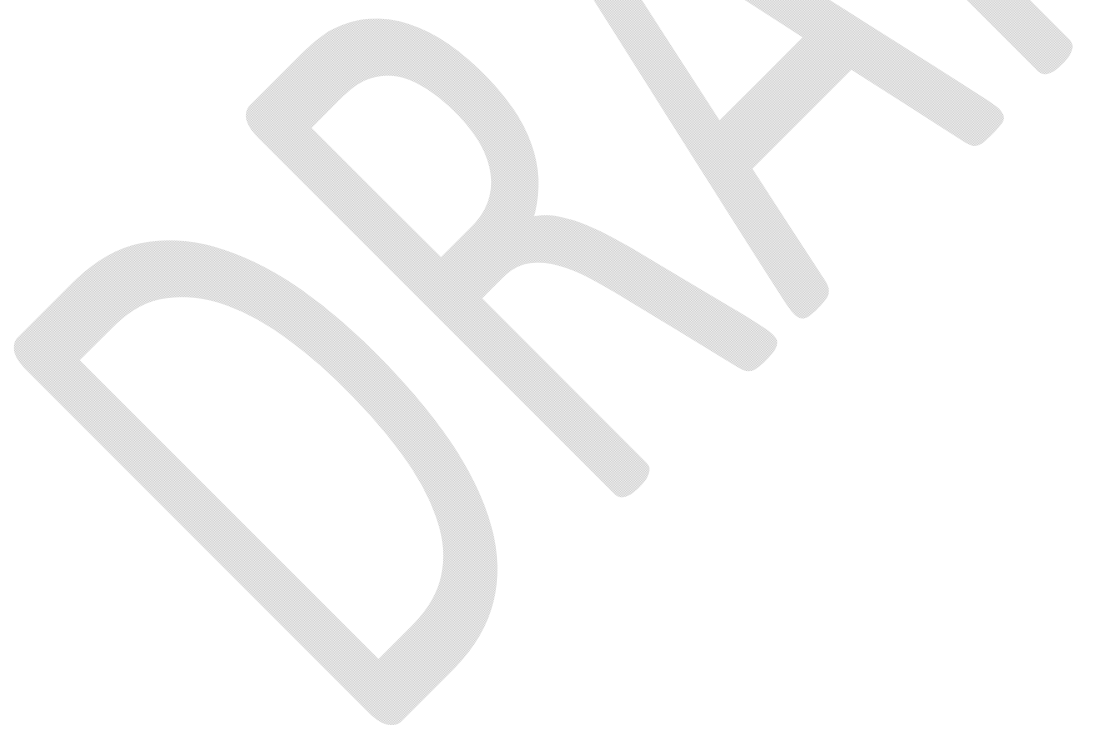




\section{References}

1. Siegel R, Naishadham D, Jemal A. Cancer statistics, 2013. Cancer J Clin 2013;63(1):1130.

2. Klotz L, Vesprini D, Sethukavalan P, et al. Long-term follow-up of a large active surveillance cohort of patients with prostate cancer. J Clin Oncol 2015;33(3):272-77.

3. Leapman MS, Cowan JE, Nguyen HG, et al. Active surveillance in younger men with prostate cancer. J Clin Oncol 2017;35(17):1898-1904.

4. van den Bergh RCN, Ahmed HU, Bangma CH, et al. Novel tools to improve patient selection and monitoring on active surveillance for low-risk prostate cancer: a systematic review. Eur Urol 2014;65(6):1023-31.

5. Carroll PR, Parsons JK, Andriole G, et al. NCCN guidelines insights: prostate cancer early detection, version 2.2016. J Natl Compr Canc Netw 2016;14(5):509-19.

6. Moore CM, Petrides N, Emberton M. Can MRI replace serial biopsies in men on active surveillance for prostate cancer? Curr Opin Urol 2014;24(3):280-7.

7. Rosenkrantz AB, Verma S, Choyke P, et al. Prostate magnetic resonance imaging and magnetic resonance imaging targeted biopsy in patients with a prior negative biopsy: a consensus statement by AUA and SAR. J Urol 2016;196(6):1613-18.

8. Fulgham PF, Rukstalis DB, Turkbey IB, et al. AUA policy statement on the use of multiparametric magnetic resonance imaging in the diagnosis, staging and management of prostate cancer. $J$ Urol 2017;198(4):832-8.

9. Weinreb JC, Barentsz JO, Choyke PL, et al. PI-RADS prostate imaging - reporting and data system: 2015, version 2. Eur Urol 2016;69(1):16-40.

10. Felker ER, Wu J, Natarajan S, et al. Serial magnetic resonance imaging in active surveillance of prostate cancer: incremental value. J Urol 2016;195(5):1421-7.

11. Habibian DJ, Liu CC, Dao A, et al. Imaging characteristics of prostate cancer patients who discontinued active surveillance on 3-T multiparametric prostate MRI. Am J Roentgenol 2017;208(3):564-9.

12. Eineluoto JT, Järvinen P, Kenttämies A, et al. Repeat multiparametric MRI in prostate cancer patients on active surveillance. PLoS One 2017;12(12).

13. Kornberg Z, Cowan JE, Westphalen AC, et al. Genomic Prostate Score, PI-RADS version 2 and progression in men with prostate cancer on active surveillance. $J$ Urol 2019;201(2):300-7.

14. Chen F, Cen S, Palmer S. Application of prostate imaging reporting and data system version 2 (PI-RADS v2): interobserver agreement and positive predictive value for localization of intermediate- and high-grade prostate cancers on multiparametric magnetic resonance imaging. Acad Radiol 2017;24(9):1101-6.

15. Turkbey B, Mani H, Shah V, et al. Multiparametric 3T prostate magnetic resonance imaging to detect cancer: histopathological correlation using prostatectomy specimens processed in customized magnetic resonance imaging-based molds. $J$ Urol 2011;186(5):1818-24.

16. Siddiqui MM, Rais-Bahrami S, Turkbey B, et al. Comparison of MR/ultrasound fusionguided biopsy with ultrasound-guided biopsy for the diagnosis of prostate cancer. JAMA 2015;313(4):390-7. 
17. An JY, Sidana A, Choyke PL, et al. Multiparametric magnetic resonance imaging for active surveillance of prostate cancer. Balkan Med J 2017;34(5):388-96.

18. Streeter EH, Brewster SF. NICE guidelines on prostate cancer active surveillance: is UK practice leading the world? BJU Int 2015;115(1):12-13.

19. Liss MA, Ehdaie B, Loeb S, et al. An update of the American Urological Association white paper on the prevention and treatment of the more common complications related to prostate biopsy. J Urol 2017;198(2):329-34.

20. Sathianathen NJ, Omer A, Harriss E, et al. Negative predictive value of multiparametric magnetic resonance imaging in the detection of clinically significant prostate cancer in the prostate imaging reporting and data system era: a systematic review and metaanalysis. Eur Urol 2020;78(3):402-14.

21. Moldovan PC, Van den Broeck T, Sylvester R, et al. What is the negative predictive value of multiparametric magnetic resonance imaging in excluding prostate cancer at biopsy? A systematic review and meta-analysis from the European Association of Urology prostate cancer guidelines panel. Eur Urol 2017;72(2):250-66.

22. Thurtle D, Barrett T, Thankappan-Nair V, et al. Progression and treatment rates using an active surveillance protocol incorporating image-guided baseline biopsies and multiparametric magnetic resonance imaging monitoring for men with favourable-risk prostate cancer. BJU Int 2018;122(1):59-65.

23. Henderson DR, de Souza NM, Thomas K, et al. Nine-year follow-up for a study of diffusion-weighted magnetic resonance imaging in a prospective prostate cancer active surveillance cohort. Eur Urol 2016;69(6):1028-33.

24. Moore CM, Giganti F, Albertsen P, et al. Reporting magnetic resonance imaging in men on active surveillance for prostate cancer: the PRECISE recommendations-a report of a European School of Oncology task force. Eur Urol 2017;71(4):648-55.

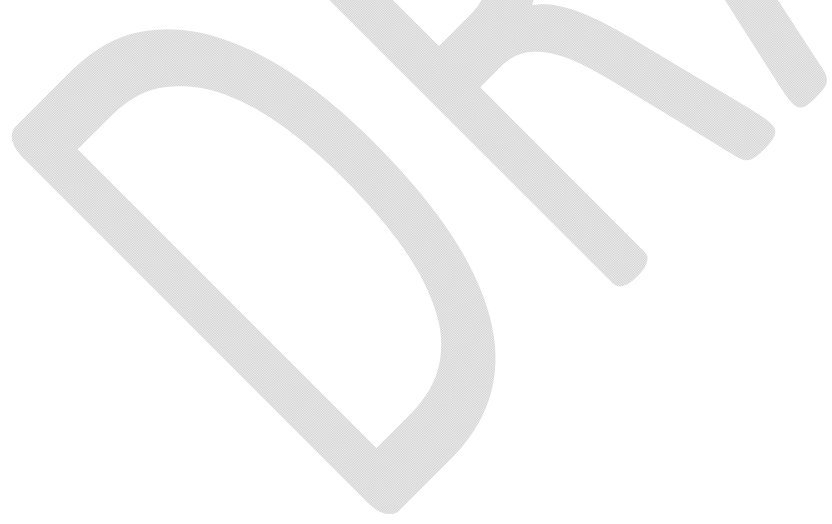




\section{Figures and Tables}

Figure 1. (A) Overall repeat magnetic resonance imaging (MRI) change from initial study, as a percentage of a total. (B) Study schema and relationship of MRI upgrade to pathological outcomes. AS: active surveillance; mp: multiparametric.

A

Upgrade

$\square$ Downgrade

$\square$ No Change

Total=121

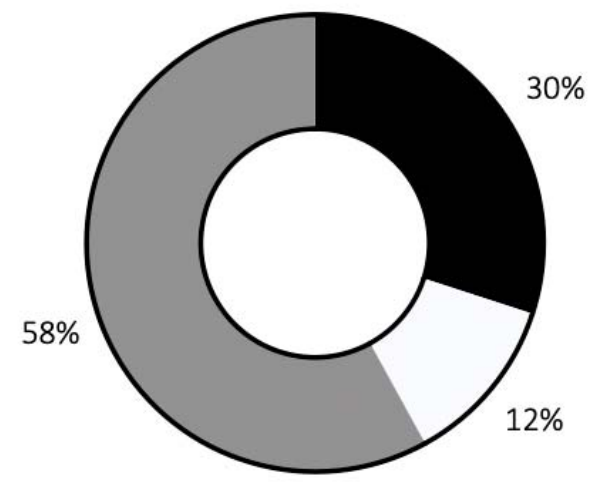

B

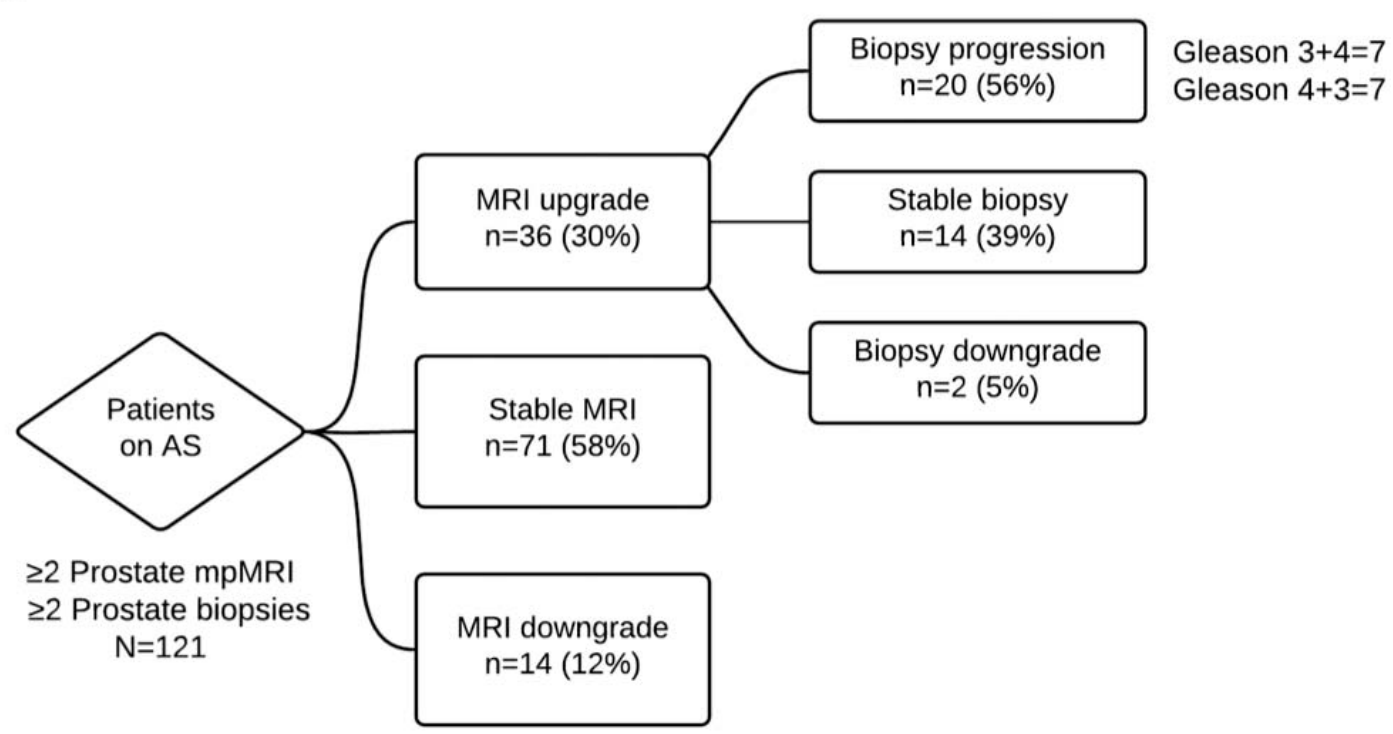


Figure 2. (A) Serial magnetic resonance imaging (MRI) upgrade test statistics. (B) Histogram of MRI upgrade and proportion of associated biopsy progression.

A

\begin{tabular}{lcc} 
& Value & 95\% Confidence Interval \\
\cline { 2 - 3 } Sensitivity & 0.364 & $0.236-0.490$ \\
Specificity & 0.758 & $0.654-0.860$ \\
Positive Predictive Value & 0.556 & $0.393-0.717$ \\
Negative Predictive Value & 0.588 & $0.483-0.692$
\end{tabular}

B

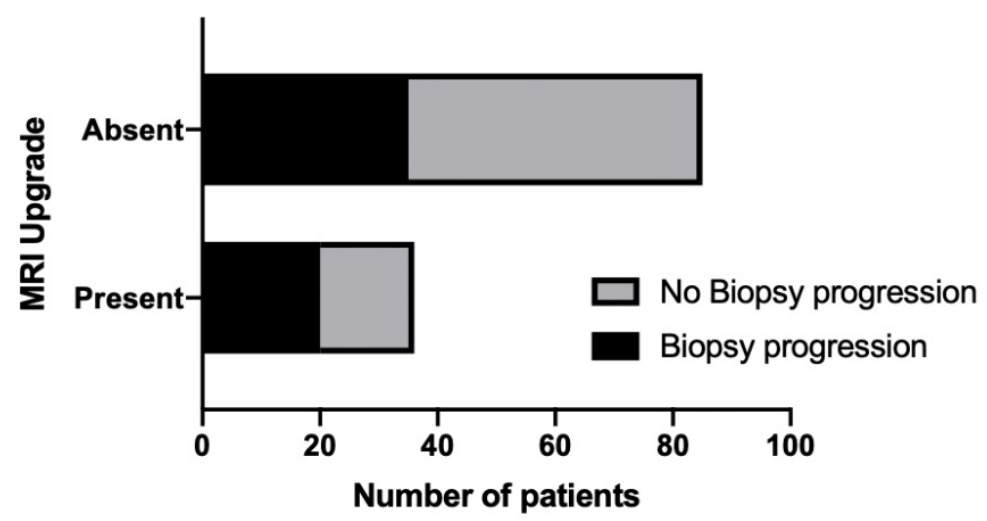

